\title{
Optical study of the vibrational and dielectric properties of $\mathrm{BiMnO}_{3}$
}

\author{
W. S. Mohamed ${ }^{1}$, A. Nucara ${ }^{2}$, G. Calestani ${ }^{3}$, F. Mezzadri ${ }^{3}$, E. Gilioli ${ }^{4}$, F. Capitani ${ }^{1}$, P. Postorino ${ }^{1}$, and P. Calvani ${ }^{2}$ \\ ${ }^{1}$ Dipartimento di Fisica, Università di Roma "La Sapienza", P.le A. Moro 2, 00185 Roma, Italy \\ ${ }^{2}$ CNR-SPIN and Dipartimento di Fisica, Università di Roma "La Sapienza", P.le A. Moro 2, 00185 Roma, Italy \\ ${ }^{3}$ Dipartimento di Chimica, Università di Parma, \\ Parco Area delle Scienze 17A, 43124 Parma, Italy and \\ ${ }^{4}$ CNR-IMEM, Parco Area delle Scienze 37A, 43124 Parma
}

(Dated: September 24, 2018)

\begin{abstract}
$\mathrm{BiMnO}_{3}(\mathrm{BMO})$, ferromagnetic $(\mathrm{FM})$ below $T_{c} \simeq 100 \mathrm{~K}$, was believed to be also ferroelectric $(\mathrm{FE})$ due to a non-centro-symmetric $\mathrm{C} 2$ structure, until diffraction data indicated that its space group is the centro-symmetric $\mathrm{C} 2 / \mathrm{c}$. Here we present infrared phonon spectra of BMO, taken on a mosaic of single crystals, which are consistent with $\mathrm{C} 2 / \mathrm{c}$ at any $T>10 \mathrm{~K}$, as well as room-temperature Raman data which strongly support this conclusion. We also find that the infrared intensity of several phonons increases steadily for $T \rightarrow 0$, causing the relative permittivity of BMO to vary from 18.5 at $300 \mathrm{~K}$ to 45 at $10 \mathrm{~K}$. At variance with $\mathrm{FE}$ materials of displacive type, no appreciable softening has been found in the infrared phonons. Both their frequencies and intensities, moreover, appear insensitive to the FM transition at $T_{c}$.
\end{abstract}

PACS numbers: 78.30.-j, 78.30.Hv, 63.20.-e

\section{INTRODUCTION}

The possible multiferroicity - or, more precisely, magnetoelectricity - of $\mathrm{BiMnO}_{3}$ (BMO), namely the simultaneous occurrence of ferroelectric (FE) and ferromagnetic (FM) long-range order in this simple perovskite, has been long discussed in the literature [1 5]. Such interest is justified by its potential applications, which may span from giant electric transformers and multiple-state memory elements, to spintronics, magnetoelectric sensors, electricfield controlled ferromagnetic devices, and variable transducers $6-11]$. Indeed, on one hand $\mathrm{BiMnO}_{3}$ is ferromagnetic below 12, 13 $T_{c} \simeq 100 \mathrm{~K}$, due to superexchange along the $\mathrm{Mn}^{3+}-\mathrm{O}^{2-} \mathrm{Mn}^{3+}$ chains, with a maximum reported magnetization of $3.92 \mu_{B}$ per formula unit [13, 14]. On the other hand, the $\mathrm{Bi}^{3+}\left(6 \mathrm{~s}^{2}\right)$ lone pair could experience a repulsion from the $2 \mathrm{p}$ orbitals of neighboring oxygen ions, leading to a permanent electric dipole and a ferroelectric distortion of the perovskite unit cell. However, both the detection of ferroelectricity, and the observation of such distortion have been long controversial, also for the difficulty to grow single crystals [15] of BMO, being it metastable at ambient pressure [14]. Indeed, the observation of ferroelectricity has been reported for thin films only [16, 17], where moreover the results strongly depend on their thickness and oxygen stoichiometry [18].

Concerning bulk BMO, it has two structural phase transitions [13], at $470 \mathrm{~K}$ from monoclinic to monoclinic, and at $770 \mathrm{~K}$ from monoclinic to orthorhombic. Both first-principle calculations [7] and an experimental study 19 supported the FE hypothesis and were consistent with early diffraction studies, which identified a noncentro-symemetric C2 space group symmetry [19, 20]. However, recent experimental studies agree that the symmetry of the whole monoclinic phase at ambient pressure is the centro-symmetric $\mathrm{C} 2 / \mathrm{c}$ [14, 21 23]. This excludes a ferroelectric phase for BMO, of the displacive type 24] at least. However, according to a recent model, inversion symmetry breaking might occur also in the $\mathrm{C} 2 / \mathrm{c}$ structure, due to some antiferromagnetic order hidden within canted ferromagnetism [25]. Finally, it was found that, for increasing pressure, in single crystals the symmetry evolves to the monoclinic $P 2_{1} / c$ at $1 \mathrm{GPa}$, and to the orthorhombic Pnma at $6 \mathrm{GPa}$ 23].

Valuable information on the crystal symmetry of an insulator can also be provided by optical studies (Raman, and/or infrared) of its phonon spectrum. A factor group analysis gives for $\mathrm{C} 2$ the representation $29 A+31 B$, where 57 phonons are both IR and Raman active while one $\mathrm{A}$ and two $\mathrm{B}$ vibrations are acoustic. In the space group C2/c, instead, the representation is $14 A_{g}+14 A_{u}$ $+16 B_{g}+16 B_{u}$ where, due to the inversion symmetry, all the $A_{g}$ and $B_{g}$ modes are Raman active, the $13 A_{u}$ and $14 B_{u}$ modes are infrared active, and the remaining ones are acoustic. Previous infrared measurements of the BMO phonon spectrum [26] were fit to the sum of 32 Lorentzian modes. This number is higher than that predicted for the $\mathrm{C} 2$ /c space group (27), even if still much lower than that expected for the C2 symmetry (57).

To check and possibly solve this apparent contradiction we have investigated the far-infrared reflectivity of a $\mathrm{BiMnO}_{3}$ mosaic of small single crystals between 10 and $300 \mathrm{~K}$. The resulting optical conductivity could be very well fit at $10 \mathrm{~K}$ to a sum of 25 Lorentzians, thus reconciling the results of infrared spectroscopy with a centrosymmetric structure for BMO. Moreover, we have taken Raman spectra on a single crystal belonging to the same mosaic, finding out that most of the 16 lines observed therein do not coincide with the infrared ones. This provides further support to centro-symmetric structure $\mathrm{C} 2 / \mathrm{c}$, which is also consistent with the observation that 
no infrared line softens appreciably for $T \rightarrow 0$, as it would occur in displacive ferroelectrivity. Finally, we observe a remarkable increase in the infrared oscillator strength for lowering temperature, which causes the extrapolated relative premittivity to increase smoothly from 18.5 at 300 $\mathrm{K}$ to 45 at $10 \mathrm{~K}$.

\section{EXPERIMENT AND RESULTS}

The $\mathrm{BiMnO}_{3}$ sample was grown and characterized as described in Ref. 23. The compound was synthesized by solid state reaction of $\mathrm{Bi}_{2} \mathrm{O}_{3}$ (Aldrich $99.99 \%$ ) and $\mathrm{Mn}_{2} \mathrm{O}_{3}$ (Aldrich $99.999 \%$ ), with the latter one in slight excess, at $4 \mathrm{GPa}$ and $1073 \mathrm{~K}$, using a high-pressure multianvil apparatus to stabilize the phase. We thus obtained a mosaic, whose dimensions are about $5 \times 4 \times 1.5 \mathrm{~mm}$, made of single crystals too small to be used individually for the far-infrared measurements. Some of them were then extracted to perform the structural and magnetic measurements, while the rest of the batch was polished with powders having grain size down to $0.5 \mu \mathrm{m}$ for the infrared experiment. Typical magnetization curves in field cooling (FC, $100 \mathrm{Oe}$ ) and zero-field cooling (ZFC) are shown in Fig. 1. They display a single and sharp FM transition at $100 \mathrm{~K}$, which demonstrates the good chemical quality of the sample.

The sample reflectivity $R(\omega)$ was measured by an interferometer Bruker $66 \mathrm{~V}$, coupled to an Infrared Labs. liquid-He cooled bolometer, a $\mathrm{Hg}$-Cd-Te detector, or a Si photodiode, depending on the spectral range, with a spectral resolution of $2 \mathrm{~cm}^{-1}$ in the phonon region. The radiation was unpolarized: even if the whole sample were a single crystal, the monoclinic structure of BMO would prevent to obtain more meaningful infor-

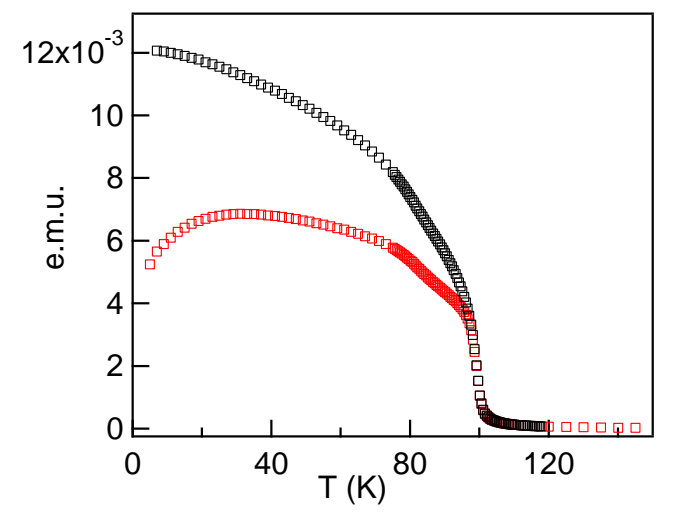

FIG. 1: Color online. Magnetization vs. temperature (in electromagnetic units, emu) of a single crystal extracted from the mosaic used for the infrared reflectivity measurements. The sample was cooled either in zero field (ZFC) or in a field of 100 Oersted (FC). A sharp and single FM transition occurs at $T_{c}=100 \mathrm{~K}$. mation by polarized radiation. The sample temperature was regulated between 10 and $300 \mathrm{~K}$ with a thermal stability and accuracy of $\pm 2 \mathrm{~K}$. The reference was a golden mirror close to the sample and oriented by a He-Ne laser parallel to it. Even if the region of interest is limited here to $50 \div 700 \mathrm{~cm}^{-1}$, the spectral range was extended from 30 to $10000 \mathrm{~cm}^{-1}$ in order to obtain accurate Kramers-Kronig (KK) transformations [27]. The extrapolation from $\omega=30 \mathrm{~cm}^{-1}$ to $\omega=0$ was performed by a constant $R(\omega)$, as usual for good insulators, that to $\omega=\infty$ by a $\omega^{-4}$ power law 28]. The Raman spectra were measured with a Horiba LabRAM HR Evolution microspectrometer in backscattering geometry. The samples were different small crystals belonging to an unpolished area of the same mosaic where the infrared spectra were measured. They were excited by the $632.8 \mathrm{~nm}$ radiation of a He-Ne laser with $30 \mathrm{~mW}$ output power. Also the Raman spectra, as the infrared ones, were taken in unpolarized configuration. The detector was a Peltiercooled Charge-Coupled Device (CCD) and the resolution $1 \mathrm{~cm}^{-1}$, thanks to a 1800 grooves/mm grating with 800 $\mathrm{mm}$ focal length. Measurements were performed with a 20x objective (numerical aperture $\mathrm{NA}=0.35$ ).

The reflectivity of $\mathrm{BiMnO}_{3}$ is shown in Fig. 2 in the far infrared range at all temperatures and is basically similar to that reported in Ref. 26, including a strong increase of the intensity of some lines for $T \rightarrow 0$. Above that range, $R(\omega)$ looks flat and basically independent of temperature within the errors.

The optical conductivity $\sigma(\omega)$ extracted by the KK transformations from $R(\omega)$ is shown in Fig. 3. The phonon parameters were obtained by fitting $\sigma(\omega)$ to the sum of Lorentzian oscillators

$$
\sigma_{1}(\omega)=\frac{1}{60} \sum_{j=1}^{n} \frac{\omega^{2} \Gamma_{j} S_{j}}{\left(\Omega_{j}^{2}-\omega^{2}\right)^{2}+\omega^{2} \Gamma_{i}^{2}}
$$

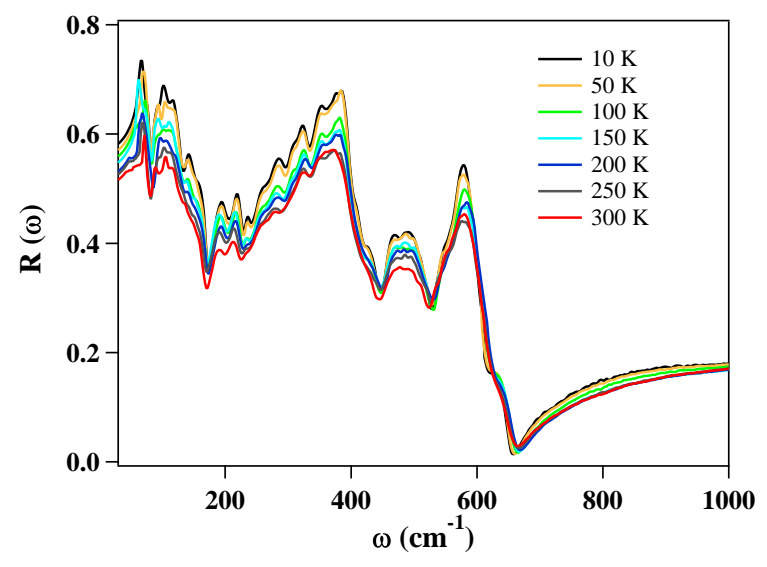

FIG. 2: Color online. Reflectivity spectra of bulk $\mathrm{BiMnO}_{3}$, in the far-infrared range of frequencies at different temperatures. 
where the factor $1 / 60$ allows for $\sigma_{1}(\omega)$ being measured [29] in $\Omega^{-1} \mathrm{~cm}^{-1}$. Here, $\Omega_{j}$ and $\Gamma_{j}$ are the peak frequency and the linewidth of the $j$-th transverse optical mode, respectively, in $\mathrm{cm}^{-1}$, and $S_{j}$ is the oscillator strength in $\mathrm{cm}^{-2}$. The fitting parameters are listed in Table I for both the highest and the lowest temperature. Excellent fits (like that the inset of Fig. 3) were obtained at 10,100 , and $150 \mathrm{~K}$ for $n=25$ oscillators, confirming the diffraction result that no structural change is associated with the FM transition. At $300 \mathrm{~K}$, due to line broadening which superimposes some modes, only 21 oscillators were necessary. As already mentioned, in Ref. 26, the infrared phonon spectrum was fit using instead $n=32$ modes, a value not compatible with the centrosymmetric $\mathrm{C} 2 / \mathrm{c}$. This discrepancy was tentatively explained by the authors in terms of overtones or combination bands - which however should have intensities much lower than the main lines, for the usual values of the anharmonic potential. The fact that here we get accurate fits down to $30 \mathrm{~cm}^{-1}$ for $n=25$, a number of modes smaller than the 27 predicted for the centro-symmetric structure $\mathrm{C} 2 / \mathrm{c}$, makes our results consistent with those of diffraction [14, 21, 22].

According to well known selection rules, the modes which are infrared-active in a centro-symmetric structure are not Raman active, and vice versa. Therefore, to further check the BMO cell symmetry, we examined the Raman spectra of three micro-crystals, which gave the same results. The experiment was done at room temperature, considering that diffraction data exclude phase transitions below $300 \mathrm{~K}$ 23], and that neither the Raman lines in Ref. 22, nor the infrared modes in Table I display

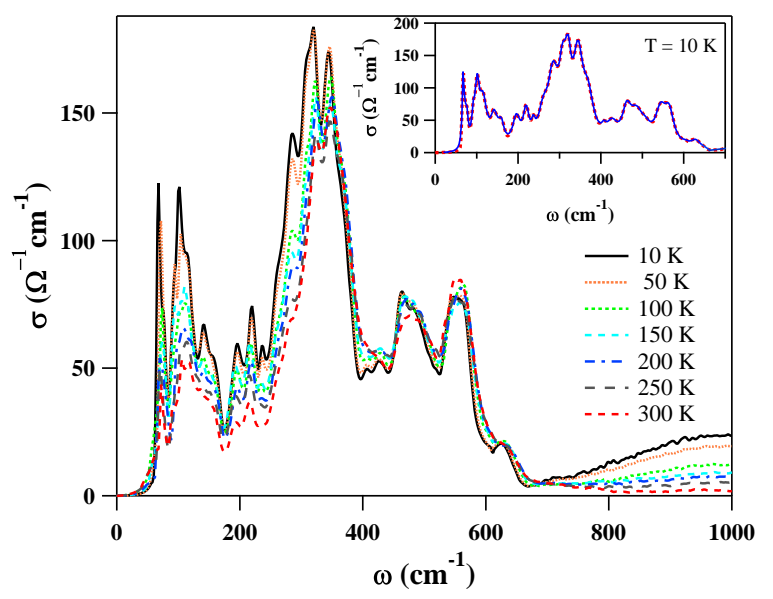

FIG. 3: Color online. Optical conductivity of bulk $\mathrm{BiMnO}_{3}$ in the far infrared range, at different temperatures. The inset shows the fit (dotted line) to the optical conductivity data (solid line) used to obtain the parameters at $10 \mathrm{~K}$ listed in Table I.
TABLE I: Infrared phonon frequencies $\Omega_{j}$, oscillator strengths $S_{j}$, and widths $\Gamma_{j}$, as obtained by fitting to Eq. (1) the experimental $\sigma(\omega)$ at $10 \mathrm{~K}$ and $300 \mathrm{~K}$. All frequencies and widths are in $\mathrm{cm}^{-1}$, intensities in $\mathrm{cm}^{-2}$.

\begin{tabular}{|c|c|c|c|c|c|c|}
\hline$j$ & $\Omega_{j}(10 \mathrm{~K})$ & $S_{j}(10 \mathrm{~K})$ & $\Gamma_{j}(10 \mathrm{~K})$ & $\Omega_{j}(300 \mathrm{~K})$ & $S_{j}(300 \mathrm{~K})$ & $\Gamma_{j}(300 \mathrm{~K})$ \\
\hline 1 & 68 & 39000 & 6 & 72 & 19000 & 8 \\
\hline 2 & 75 & 30000 & 10 & & & \\
\hline 3 & 92 & 21000 & 9 & 92 & 20000 & 16 \\
\hline 4 & 101 & 60000 & 12 & 105 & 16000 & 11 \\
\hline 5 & 115 & 100000 & 23 & 117 & 50000 & 24 \\
\hline 6 & 143 & 54000 & 21 & 139 & 44000 & 31 \\
\hline 7 & 159 & 26000 & 16 & 157 & 19000 & 20 \\
\hline 8 & 196 & 57000 & 22 & 192 & 17000 & 18 \\
\hline 9 & 219 & 49000 & 17 & 216 & 32000 & 24 \\
\hline 10 & 237 & 12000 & 10 & 235 & 3000 & 12 \\
\hline 11 & 261 & 78000 & 29 & 260 & 39000 & 32 \\
\hline 12 & 284 & 178000 & 30 & 280 & 42000 & 31 \\
\hline 13 & 308 & 115000 & 22 & 304 & 76000 & 38 \\
\hline 14 & 321 & 90000 & 18 & 321 & 80000 & 24 \\
\hline 15 & 345 & 230000 & 30 & 346 & 120000 & 50 \\
\hline 16 & 369 & 110000 & 31 & 371 & 100000 & 44 \\
\hline 17 & 407 & 7500 & 12 & & & \\
\hline 18 & 426 & 56000 & 34 & 421 & 40000 & 46 \\
\hline 19 & 463 & 80000 & 28 & 462 & 39000 & 30 \\
\hline 20 & 486 & 110000 & 35 & 489 & 60000 & 45 \\
\hline 21 & 508 & 48000 & 38 & & & \\
\hline 22 & 541 & 47000 & 22 & 545 & 46000 & 25 \\
\hline 23 & 561 & 125000 & 35 & 565 & 122000 & 39 \\
\hline 24 & 604 & 3000 & 46 & & & \\
\hline 25 & 628 & 18000 & 26 & 629 & 10000 & 28 \\
\hline
\end{tabular}

major frequency shifts down to the lowest temperatures. A typical Raman spectrum is shown in Fig. 4, where sixteen lines are observed, out of the predicted thirty. Three out of them, at or above $400 \mathrm{~cm}^{-1}$, are poorly resolved. The data are very similar to those reported for room temperature in Ref. 22, but thanks to a somewhat larger experimental range we observe an additional sharp line at $51 \mathrm{~cm}^{-1}$. In the same Fig. 4 we report also the best resolved infrared spectrum of the mosaic in Fig. 3, that at $10 \mathrm{~K}$. The two spectra look quite different and, except for a few features where the finite linewidths do not allow for a certain claim, the Raman lines do not coincide with the infrared ones. The Raman phonon frequencies and widths, obtained by fitting to data a sum of Lorentzians, are listed in Table II, which allows also for a comparison with the IR frequencies in Table I at $300 \mathrm{~K}$. These results provide further support to our previous conclusion that the ions of BMO vibrate in a centro-symmetric cell.

Another interesting feature of the spectra in Fig. 3 is their temperature dependence. The frequencies do not change appreciably with $T$, and no phonon softening is 
observed which may indicate displacive ferroelectricity not even incipient as in $\mathrm{SrTiO}_{3}$ [30]. Moreover, we do not observe any appreciable shift in the $\omega_{j}$ 's around or below the FM transition. In Ref. 22, a low-frequency shift was reported for the Raman bands at $513.8 \mathrm{~cm}^{-1}$ and 637.4 $\mathrm{cm}^{-1}$ below $T_{c}$. The authors attribute this observation to a spin-phonon coupling which is switched on by the FM transition. The absence of a corresponding effect in the present infrared data indicates that such interaction, if may be able to change the Raman polarizability, does not affect appreciably the first-order interaction of the radiation with the lattice dipole moment.

However, as we already mentioned and is evident in Table I, the oscillator strengths $S_{j}$ of some lines (with $j$ $=1,4,5,8,11,12,15,20,25)$ increase by about a factor of two, or even more, from 300 to $10 \mathrm{~K}$, an effect that can be observed also in Fig. 2 of Ref. 26. Fits to our data at all temperatures show that the intensity of those phonons linearly increases for decreasing temperature, as shown in Fig. 5] and is insensitive to the FM transition at $T_{c}$. Similar effects have been observed in several insulating oxides with high polarizability [32 35] and often attributed to the so called "charged phonon" effect 31] by which phonons acquire an anomalously large spectral weight due to a redistribution of electronic charge, within the cell, associated with their displacement pattern. Such increase of spectral weight in the far-infrared region causes the failure of the $f$-sum rule

$$
\int_{0}^{\omega_{\max }} \sigma(\omega, T) d \omega=\text { const. }
$$

if $\omega_{\max }$ does not include the regions where the trans-

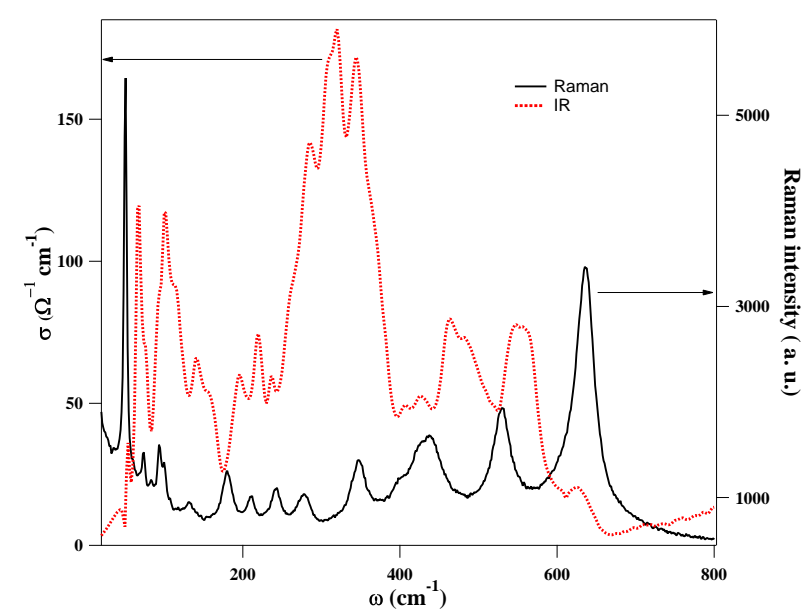

FIG. 4: Color online. Raman spectrum of a single crystal of $\mathrm{BiMnO}_{3}$ at room temperature and infrared spectrum of the mosaic at $10 \mathrm{~K}$, extracted from Fig. 3. Given the weak $T$ dependence observed in both spectra (see Table I and Ref. 22) the best resolved IR spectrum has been chosen for a more meningful comparison. ferred spectral weight comes from, presumably the electronic bands in the visible and the UV, which are out of the present measuring range. Anyway, checking such assumption would be a very hard task, given the small width of the FIR range (where the spectral weight in Eq. 2 increases for lowering temperature) with respect of the spectral regions where it should decrease. The same holds for the weak band partially shown in Fig. 3 , at frequencies higher than the phonon range. It can either result from an admixture of phonon overtones and combination bands, consistently with its low intensity, or have a polaronic origin, namely, to come from excess charges self-trapped in the lattice. Those charges could be related to oxygen non-stoichiometry, which is likely to occur in this oxide [36]. In both cases the band intensity will increase for $\mathrm{T} \rightarrow 0$ : in the former one because it will follow the strengthening of the individual phonons, in the latter one because, at low $T$, more quasi-free charges will self-trap in the local distortions [37, 38].

Together with the optical conductivity, which is related to the imaginary part of the dielectric function $\tilde{\epsilon}(\omega)$, the KK transformations provide its real part $\epsilon_{1}(\omega)$, that is shown in Fig. 6. If one assumes the absence of strong modes at frequencies lower than the limit of this experiment $\left(30 \mathrm{~cm}^{-1}\right)$, its extrapolation to zero frequency gives the relative permittivity $\epsilon_{1}(0)$, which can be thus be determined vs. temperature without any metal deposition and contacts on the sample, which may affect the measurement. As shown in the inset of the same Figure, due to the above described growth of several phonon intensi-


FIG. 5: Color online. Systematic increase of the oscillator strength of $\mathrm{BiMnO}_{3}$ phonons for $T \rightarrow 0$. The modes (M) are numbered as in Table I and the lines are guides to the eye. Within errors, no reproducible features are observed around the FM transition at $T_{c} \simeq 100 \mathrm{~K}$. 
TABLE II: Raman phonon frequencies $\Omega_{j}$ obtained by fitting to Eq. (1) the $\mathrm{BiMnO}_{3}$ Raman intensity at $300 \mathrm{~K}$. All frequencies and widths are in $\mathrm{cm}^{-1}$.

\begin{tabular}{ccc}
\hline \hline Phonon $(j)$ & $\Omega_{j}(300 \mathrm{~K})$ & $\Gamma_{j}(300 \mathrm{~K})$ \\
\hline 1 & 50 & 2 \\
2 & 74 & 1 \\
3 & 84 & 1 \\
4 & 93 & 1 \\
5 & 99 & 1 \\
6 & 133 & 6 \\
7 & 180 & 9 \\
8 & 211 & 9 \\
9 & 242 & 10 \\
10 & 278 & 15 \\
11 & 347 & 15 \\
12 & 400 & 15 \\
13 & 424 & 12 \\
14 & 440 & 17 \\
15 & 530 & 12 \\
16 & 635 & 14 \\
& & \\
\hline \hline
\end{tabular}

ties at low $T$, it increases smoothly from 18.5 at $300 \mathrm{~K}$ to 45 at $10 \mathrm{~K}$. These rather low values support once again the absence of a ferroelectric phase in $\mathrm{BiMnO}_{3}$ down to $10 \mathrm{~K}$.

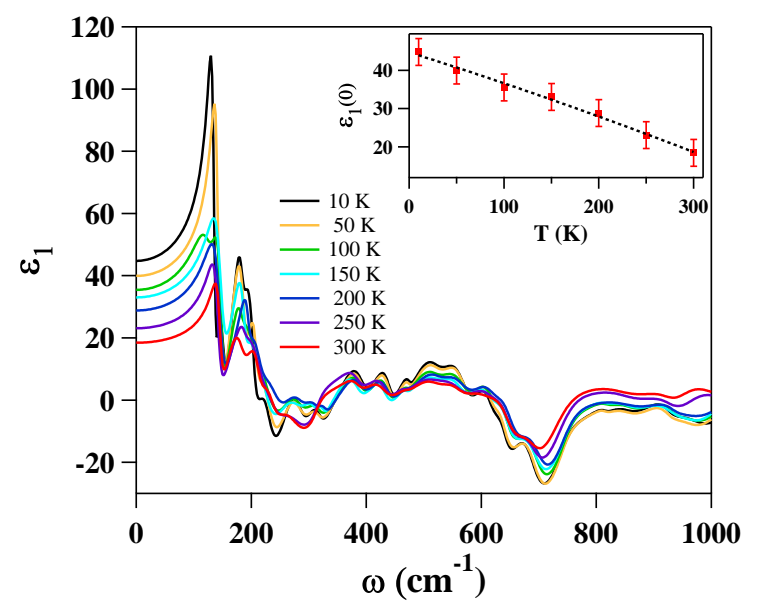

FIG. 6: Color online. Behavior with frequency and temperature of the real part of the dielectric function $\epsilon_{1}(\omega)$ in $\mathrm{BiMnO}_{3}$. The inset shows the temperature dependence of its extrapolation to zero frequency, the (relative) dielectric constant $\epsilon_{1}(0)$.

\section{CONCLUSION}

In order to further investigate the possible occurrance of ferroelectricity in $\mathrm{BiMnO}_{3}$, we have measured the infrared phonon spectrum of a mosaic of small single crystals from 300 to $10 \mathrm{~K}$. We find that at the lowest $T$ it can be accurately fit by using only 25 Lorentz oscillators, out of the 27 predicted for the $\mathrm{C} 2 /$ c symmetry that has been attributed to $\mathrm{BiMnO}_{3}$ by the most recent diffraction experiments. To further check the conclusion that the $\mathrm{BMO}$ cell is centro-symmetric we have measured the Raman spectrum at $300 \mathrm{~K}$ of three single crystals belonging to the mosaic, and we have observed sixteen phonon lines out of the thirty predicted for $\mathrm{C} 2 / \mathrm{c}$. Most of them are definitely different from the infrared lines, while for a few others, the finite linewidths in both spectra do not allow for an unambiguous conclusion. This is consistent with a centro-symmetric structure like $\mathrm{C} 2 / \mathrm{c}$ while, in case of broken inversion symmetry, the Raman and infrared combs should substantially coincide. If one adds that our data do not show any appreciable phonon softening, the present results point consistently toward the absence of ferroelectricity - and therefore of multiferroicity - in $\mathrm{BiMnO}_{3}$ down to $10 \mathrm{~K}$, at least of displacive type.

However, we observe a regular increase of the oscillator strength in several modes of $\mathrm{BiMnO}_{3}$ for lowering temperature. This causes the extrapolation to zero frequency of the real part of the dielectric function, namely, the relative permittivity, to increase from 18.5 at $300 \mathrm{~K}$ to 45 at $10 \mathrm{~K}$. Across the ferromagnetic transition at $T_{c} \simeq$ $100 \mathrm{~K}$ we do not detect any major anomaly in the oscillator strength, nor in the phonon frequency. Therefore, we conclude that the phonon-spin interaction recently invoked to interpret the behavior of a few Raman phonon lines does not affect the dipole moment which interacts at first order with the radiation field. 
[1] N. Hur, S. Park, P. A. Sharma, S. Guha, and S.-W. Cheong, Phys. Rev. Lett. 93, 107207 (2004).

[2] L. C. Chapon, G. R. Blake, M. J. Gutmann, S. Park, N. Hur, P. G. Radaelli, and S.-W. Cheong, Phys. Rev. Lett. 93, 177402 (2004).

[3] M. Fiebig, Th. Lottermoser, D. Frohlich, A. V. Goltsev, and R. V. Pisarev, Nature (London) 419, 818 (2002).

[4] M.Grizalez, G.A.Mendoza, and P.Prieto, J. Phys.: conf. series 167, 012035 (2009).

[5] Th. Lottermoser, Th. Lonkai, U. Amann, D. Hohlwein, J. Ihringer, and M. Fiebig, Nature (London) 430, 541 (2004).

[6] C. Binek and B. Doudin, J. Phys.: Condens. Matter 17, L39 (2005).

[7] N. A. Hill and K. M. Rabe, Phys. Rev. B59, 8759 (1999).

[8] W. Eerenstein, N. D. Mathur, and J. F. Scott, Nature (London) 442, 759 (2006).

[9] A.A.Belik,J.Solid State Chem. 195, 32 (2012).

[10] M. Fiebig, J. Phys. D 38, R123 (2005).

[11] S. W. Cheong and M. Mostovoy, Nat. Mater. 6,13 (2007).

[12] F. Sugawara, S. Iiida,Y. Syono, and S. Akimoto, J. Phys. Soc. Jpn. 25,1553 (1968).

[13] T. Kimura, S. Kawamoto, I. Yamada, M. Azuma, M. Takano and Y. Tokura, Phys. Rev. B 67, 180401(R) (2003).

[14] E. Montanari, G. Calestani, L. Righi, E. Gilioli, F. Bolzoni, K S. Knight and P G. Radaelli, Phys. Rev. B 75, 220101 (2007).

[15] P. Toulemonde, C. Darie, C. Goujon, M. Legendre, T. Mendonca, M. lvarez-Murga, V. Simonet, P. Bordet, P. Bouvier, J. Kreisel, and M. Mezouar, High Press. Res. 29, 600 (2009).

[16] A. Moreira dos Santos, S. Parashar, A. R. Raju, Y. S. Zhao, A. K. Cheetham and C. N. R. Rao, Solid State Commun. 122, 49 (2002).

[17] A. Sharan, J. Lettieri, Y. F. Jia, W. Tian, X. Q. Pan, D. G. Schlom, and V. Gopalan, Phys. Rev. B 69, 214109 (2004).

[18] E. Montanari, L. Righi, G. Calestani, A. Migliori, E. Gilioli, F. Bolzoni, Chem. Mater. 17, 1765 (2005).

[19] A. M. dos Santos, A. K. Cheetham, T. Atou, Y. Syono, Y. Yamaguchi, K. Ohoyama, H. Chiba, and C. N. R. Rao, Phys. Rev. B 66, 064425 (2002).

[20] T. Atou, H. Chiba, K. Ohoyama, Y. Yamaguchi, and Y.
Syono, J. Solid State Chem.145, 639 (1999).

[21] A. A. Belik, S. Iikubo, T. Yokosawa, K. Kodama, N. Igawa, S. Shamoto, M. Azuma, M. Takano, K. Kimoto, Y. Matsui and E. Takayama-Muromachi J. Am. Chem. Soc. 129, 971 (2007).

[22] P. Toulemonde, P. Bordet, P. Bouvier, and J. Kreisel Phys. Rev. B 89, 224107 (2014).

[23] G. Calestani, F. Orlandi, F. Mezzadri, L. Righi, M.Merlini, and E. Gilioli, Inorg. Chem. 53, 8749 (2014).

[24] J. F. Scott, Rev. Mod. Phys. 46, 83 (1974).

[25] I. V. Solovyev and Z. V. Pchelkina, Phys. Rev. B 82, 094425 (2010).

[26] V. Goian, S. Kamba, M. Savinov, D. Nuzhnyy, F. Borodavka, P. Vank, and A. A. Belik, J. Appl. Phys. 112, 074112 (2012).

[27] M. Dressel and G. Grüner, Electrodynamics of Solids, Cambridge Univ. Press, Cambridge (UK), 2002, p. 56.

[28] J. Yang, J. Hwang, T. Timusk, A. S. Sefat, and J. E. Greedan, Phys. Rev. B 73, 195125 (2006).

[29] T. Timusk and T. Tanner, Infrared properties of high $T_{c}$ superconductors, p. 347, in Physical properties og high $T_{c}$ superconductors, ed. by D. M. Ginsberg, World Scientific, Singapore, 1989.

[30] A. Yamanaka, M. Kataoka, Y. Inaba, K. Inoue, B. Hehlen, and E. Courtens, Europhys. Lett. 50, 688 (2000), and References therein.

[31] M. J. Rice, Solid State Commun. 31, 93 (1979).

[32] C. C. Homes, T. Vogt, S. M. Shapiro, S. Wakimoto, and A. P. Ramirez, Science 293, 673 (2001).

[33] C. C. Homes, T. Vogt, S. M. Shapiro, S. Wakimoto, M. A. Subramanian, and A. P. Ramirez, Phys. Rev. B 67, 092106 (2003).

[34] P. Calvani, M. Capizzi, S. Lupi, and G. Balestrino, EPL 31, 473 (1995).

[35] A. Nucara, W. S. Mohamed, L. Baldassarre, S. Koval, J. Lorenzana, R. Fittipaldi, G. Balakrishnan, A. Vecchione, and P. Calvani, Phys. Rev. B 90, 014304 (2014).

[36] D. W. Boukhvalov and I. V. Solovyev, Phys. Rev. B 82, 245101 (2010).

[37] A. J. Millis, R. Mueller, and Boris I. Shraiman, Phys. Rev. B 54, 5405 (1996).

[38] P. Calvani, Riv. Nuovo Cimento 24, 1 (2001). 